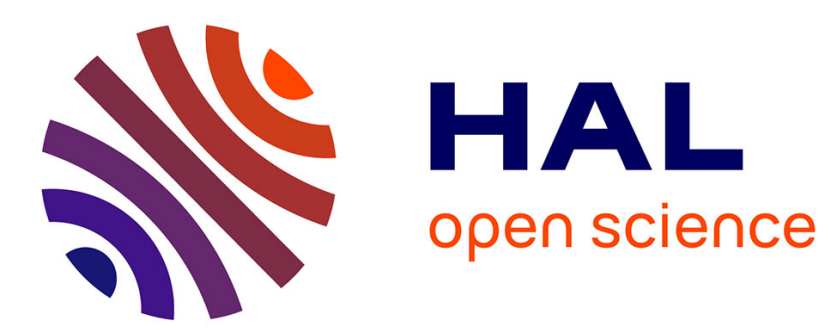

\title{
Optimal Parameter Estimation in Heterogeneous Clutter for High Resolution Polarimetric SAR Data
}

Gabriel Vasile, Frédéric Pascal, Jean-Philippe Ovarlez, Pierre Formont, Michel Gay

\section{- To cite this version:}

Gabriel Vasile, Frédéric Pascal, Jean-Philippe Ovarlez, Pierre Formont, Michel Gay. Optimal Parameter Estimation in Heterogeneous Clutter for High Resolution Polarimetric SAR Data. IEEE Geoscience and Remote Sensing Letters, 2011, 8 (6), pp.1046-1050. 10.1109/LGRS.2011.2152363 . hal-00638825

\section{HAL Id: hal-00638825 https://hal.science/hal-00638825}

Submitted on 7 Nov 2011

HAL is a multi-disciplinary open access archive for the deposit and dissemination of scientific research documents, whether they are published or not. The documents may come from teaching and research institutions in France or abroad, or from public or private research centers.
L'archive ouverte pluridisciplinaire HAL, est destinée au dépôt et à la diffusion de documents scientifiques de niveau recherche, publiés ou non, émanant des établissements d'enseignement et de recherche français ou étrangers, des laboratoires publics ou privés. 


\title{
Optimal Parameter Estimation in Heterogeneous Clutter for High Resolution Polarimetric SAR Data
}

\author{
Gabriel Vasile, Member, IEEE, Frédéric Pascal, Member, IEEE, Jean-Philippe Ovarlez, Member, IEEE, \\ Pierre Formont, Student Member, IEEE Michel Gay, Member, IEEE
}

\begin{abstract}
This paper presents a new estimation scheme for optimally deriving clutter parameters with high resolution POLSAR data. The heterogeneous clutter in POLSAR data is described by the Spherically Invariant Random Vectors model. Three parameters are introduced for the high resolution POLSAR data clutter: the span, the normalized texture and the speckle normalized covariance matrix. The asymptotic distribution of the novel span estimator is investigated. A novel heterogeneity test for the POLSAR clutter is also discussed. The proposed method is tested with airborne POLSAR images provided by the ONERA RAMSES system.
\end{abstract}

Index Terms-Estimation, detection, polarimetry, SAR.

\section{INTRODUCTION}

The recently launched polarimetric SAR (POLSAR) systems are now capable of producing high quality images of the Earth's surface with meter resolution. The goal of the estimation process is to derive the scene signature from the observed data set. In the case of spatially changing surfaces ("heterogeneous" or "textured" scenes) the first step is to define an appropriate model describing the dependency between the polarimetric signature and the observable as a function of the speckle. In general, the multiplicative model has been employed for POLSAR data processing as a product between the square root of a scalar positive quantity (texture) and the description of an equivalent homogeneous surface (speckle) [1], [2].

In the context of the non-Gaussian polarimetric clutter models, several studies tackled POLSAR parameter estimation using the product model. For deterministic texture, Novak and Burl derived the Polarimetric Whitening Filter (PWF) by optimally combining the elements of the polarimetric covariance matrix to produce a single scalar image [1], [3]. Using the complex Wishart distribution, the PWF for homogeneous surfaces has been generalized to an Multi-look PWF (MPWF) in [2], [4]. The objective of this paper is to present a novel parameter estimation technique based on the Spherically

G. Vasile and M. Gay are with the Grenoble-Image-sPeech-SignalAutomatics Lab (GIPSA-lab), CNRS, Grenoble, France (e-mail: gabriel.vasile@gipsa-lab.grenoble-inp.fr; michel.gay@gipsa-lab.grenobleinp.fr)

F. Pascal is with the Supélec, National University of Singapore, Defence Science and Technology Agency Research Alliance, SONDRA, Gif-surYvette, France (e-mail: frederic.pascal@supelec.fr)

J.-P. Ovarlez and P. Formont are with the French Aerospace Lab (ONERA), DEMR/TSI, Palaiseau, France and with the Supélec, National University of Singapore, Defence Science and Technology Agency Research Alliance, SONDRA, Gif-sur-Yvette, France (e-mail: ovarlez@onera.fr; pierre.formont@supelec.fr)
Invariant Random Vectors (SIRV) model. For a detailed review on the use of SIRV with POLSAR data refer to [5].

This paper is organized as follows. The POLSAR parameter estimation strategy for SIRV clutter model both with normalized texture, and with normalized covariance matrix is presented in Sect. П] and Sect. [II respectively. Then, the novel span estimator is introduced in Sect.IV Next, some estimation results are shown in Sect. $\nabla$ on a real high-resolution POLSAR dataset acquired by the ONERA RAMSES system. Eventually, in Sect. VI, some conclusions are presented.

\section{SIRV CLUTTER MODEL WITH NORMALIZED TEXTURE}

The SIRV is a class of non-homogeneous Gaussian processes with random variance [6], [7]. The complex $m$ dimensional measurement $\mathbf{k}$ ( $m$ being the number of polarimetric channels) is defined as the product between the independent complex circular Gaussian vector $\zeta \sim \mathcal{N}(0,[T])$ (speckle) with zero mean and covariance matrix $[T]=E\left\{\zeta \zeta^{\dagger}\right\}$ and the square root of the positive random variable $\xi$ (representing the texture): $\mathbf{k}=\sqrt{\xi} \cdot \zeta$. It is important to notice that in the SIRV definition, the probability density function (PDF) of the texture random variable is not explicitly specified. As a consequence, SIRVs describe a whole class of stochastic processes [8].

For POLSAR clutter, the SIRV product model is the product of two separate random processes operating across two different statistical axes [5]. The polarimetric diversity is modeled by the multidimensional Gaussian kernel. The randomness of spatial variations in the radar backscattering from cell to cell is characterized by $\xi$. Relatively to the polarimetric axis, the texture random variable $\xi$ can be viewed as a unknown deterministic parameter from cell to cell.

The texture and the covariance matrix unknown parameters can be estimated from the ML theory. For $N$ i.i.d. (independent and identically distributed) secondary data, let $L_{\mathbf{k}}\left(\mathbf{k}_{1}, \ldots, \mathbf{k}_{N} \mid[T], \xi_{1}, \ldots, \xi_{N}\right)$ be the likelihood function to maximize with respect to $[T]$ and $\xi_{i}$.

$$
\begin{gathered}
L_{\mathbf{k}}\left(\mathbf{k}_{1}, \ldots, \mathbf{k}_{N} ;[T], \xi_{1}, \ldots, \xi_{N}\right)=\frac{1}{\pi^{m N} \operatorname{det}\{[T]\}^{N}} \times \\
\times \prod_{i=1}^{N} \frac{1}{\xi_{i}^{m}} \exp \left(-\frac{\mathbf{k}_{i}^{\dagger}[T]^{-1} \mathbf{k}_{i}}{\xi_{i}}\right) .
\end{gathered}
$$

The corresponding ML estimators are given by [9]:

$$
\frac{\partial \ln L_{\mathbf{k}}\left(\mathbf{k}_{1}, \ldots, \mathbf{k}_{N} \mid[T], \xi_{1}, \ldots, \xi_{N}\right)}{\partial \xi_{i}}=0 \Leftrightarrow \widehat{\xi}_{i}=\frac{\mathbf{k}_{i}^{\dagger}[T]^{-1} \mathbf{k}_{i}}{m},
$$




$$
\frac{\partial \ln L_{\mathbf{k}}\left(\mathbf{k}_{1}, \ldots, \mathbf{k}_{N} \mid[T], \xi_{1}, \ldots, \xi_{N}\right)}{\partial[T]}=0 \Leftrightarrow[\widehat{T}]=\frac{1}{N} \sum_{i=1}^{N} \frac{\mathbf{k}_{i} \mathbf{k}_{i}^{\dagger}}{\widehat{\xi}_{i}}
$$

As the variables $\xi_{i}$ are unknown, the following normalization constraint on the texture parameters assures that the ML estimator of the speckle covariance matrix is the Sample Covariance Matrix (SCM):

$$
[\widehat{T}]=\frac{1}{N} \sum_{i=1}^{N} \mathbf{k}_{i} \mathbf{k}_{i}^{\dagger}=[\widehat{T}]_{S C M} \Leftrightarrow \frac{1}{N} \sum_{i=1}^{N} \mathbf{k}_{i} \mathbf{k}_{i}^{\dagger}\left(1-\frac{1}{\widehat{\xi}_{i}}\right)=\left[0_{m}\right] .
$$

The generalized ML estimator for $\xi_{i}$ are obtained by introducing Eq. 4 in Eq. 2

$$
\widehat{\xi}_{i}=\frac{\mathbf{k}_{i}^{\dagger}[\widehat{T}]_{S C M}^{-1} \mathbf{k}_{i}}{m} .
$$

Note the $\mathbf{k}_{i}$ primary data is the cell under study.

The normalized texture estimator from Eq. 5 is known as the Polarimetric Whitening Filter (PWF-SCM) introduced by Novak and Burl in [1].

\section{SIRV CLUTTER MODEL WITH NORMALIZED COVARIANCE MATRIX}

Let now the covariance matrix be of the form $[T]=\sigma_{0}[M]$, such that $\operatorname{Tr}\{[M]\}=1$. The product model can be also written as $\mathbf{k}=\sqrt{\tau} \cdot \mathbf{z}$, where $\mathbf{z} \sim \mathcal{N}(\mathbf{0},[M]) . \sigma_{0}$ and $\xi$ are two scalar positive random variables such that $\tau=\sigma_{0} \cdot \xi$.

Using the same procedure as in Sect. and given the fact that the covariance matrix is normalized, it is possible to compute the generalized ML estimator of $[M]$ as the solution of the following recursive equation:

$$
[\widehat{M}]_{F P}=f\left([\widehat{M}]_{F P}\right)=\frac{1}{N} \sum_{i=1}^{N} \frac{\mathbf{k}_{i} \mathbf{k}_{i}^{\dagger}}{\mathbf{k}_{i}^{\dagger}[\widehat{M}]_{F P}^{-1} \mathbf{k}_{i}} .
$$

This approach has been used in [10] by Conte et al. to derive a recursive algorithm for estimating the matrix $[M]$. This algorithm consists in computing the Fixed Point of $f$ using the sequence $\left([M]_{i}\right)_{i \geq 0}$ defined by:

$$
[M]_{i+1}=f\left([M]_{i}\right) .
$$

This study has been completed by the work of Pascal et al. [11], [12], which recently established the existence and the uniqueness, up to a scalar factor, of the Fixed Point estimator of the normalized covariance matrix, as well as the convergence of the recursive algorithm whatever the initialization. The algorithm can therefore be initialized with the identity matrix $[\widehat{M}]_{0}=\left[I_{m}\right]$.

The generalized ML estimator (PWF-FP) for the $\tau_{i}$ texture for the primary data $\mathbf{k}_{i}$ is given by:

$$
\widehat{\tau}_{i}=\frac{\mathbf{k}_{i}^{\dagger}[\widehat{M}]_{F P}^{-1} \mathbf{k}_{i}}{m} .
$$

One can observe that the PWF-FP texture from Eq. 8 has the same form as the PWF-SCM. The only difference is the use of the normalized covariance estimate given by the FP estimator instead of the conventional SCM [5].

\section{MAIN RESULT}

The span (total power) $\sigma_{0}$ can be derived using the covariance matrix estimators presented in Sect. [I and Sect. [II as:

$$
\widehat{\sigma_{0}}=\frac{\mathbf{k}^{\dagger}[\widehat{M}]_{F P}^{-1} \mathbf{k}}{\mathbf{k}^{\dagger}[\widehat{T}]_{S C M}^{-1} \mathbf{k}} \text {. }
$$

Note that Eq. 9 is valid when considering $N$ identically distributed linearly independent secondary data and one primary data. It can be seen as a double polarimetric whitening filter issued from two equivalent SIRV clutter models: with normalized texture variables and with normalized covariance matrix parameter.

The main advantage of the proposed estimation scheme is that it can be directly applied with standard boxcar neighborhoods.

\section{A. Asymptotic statistics of $\widehat{\sigma_{0}}$}

This section is dedicated to the study of large sample properties and approximations of the span estimator $\widehat{\sigma_{0}}$ form Eq. 9

On one hand, the asymptotic distribution of the FP estimator from Eq. 6 has been derived in [12]. The FP estimator computed with $N$ secondary data converges in distribution to the normalized SCM computed with $N[m /(m+1)]$ secondary data. Since the normalized SCM is the SCM up to a scale factor, we may conclude that, in problems invariant with respect to a scale factor on the covariance matrix, the FP estimate is asymptotically equivalent to the SCM computed with $N[m /(m+1)]$ secondary data. Hence one can set the degrees of freedom of FP normalized covariance matrix estimators as:

$$
q_{1}=N \frac{m}{m+1} .
$$

On the other hand, Chatelain et al. established the multisensor bivariate gamma distribution PDF, whose margins are univariate gamma distributions with different shape parameters [13]:

$$
P_{b \Gamma}\left(y_{1}, y_{2} ; p_{1}, p_{2}, p_{12}, q_{1}, q_{2}\right) .
$$

The scale parameters $p_{2}$ and $p_{1}$, the shape parameters $q_{2}>$ $q_{1}$ and $p_{12}$ are linked to the mean parameters $\mu_{1}, \mu_{2}$, to the number of degrees of freedom $n_{1}, n_{2}$, and to the normalized correlation coefficient $\rho$ such as:

$q_{1}=n_{1}, q_{2}=n_{2}, p_{1}=\frac{\mu_{1}}{q_{1}}, p_{2}=\frac{\mu_{2}}{q_{2}}, p_{12}=\frac{\mu_{1} \mu_{2}}{q_{1} q_{2}}(1-\rho)$.

Using these results, we derived the PDF of the ratio $R=$ $y_{1} / y_{2}$ of two correlated Gamma random variables:

$$
\begin{gathered}
P_{R \Gamma}\left(R, p_{1}, p_{2}, p_{12}, q_{1}, q_{2}\right)=R^{q_{1}-1}\left(\frac{p_{2}}{p_{12}}\right)^{q_{1}}\left(\frac{1}{p_{2}}\right)^{q_{2}} \times \\
\times\left(\frac{p_{12}}{p_{1}+R p_{2}}\right)^{q_{2}+q_{1}} \frac{\Gamma\left(q_{1}+q_{2}\right)}{\Gamma\left(q_{1}\right) \Gamma\left(q_{2}\right)} \times \\
\times \mathbf{H}_{3}\left[q_{1}+q_{2}, q_{2}-q_{1}, q_{2} ; R \frac{p_{1} p_{2}-p_{12}}{\left(p_{1}+R p_{2}\right)^{2}}, \frac{p_{1} p_{2}-p_{12}}{p_{2}\left(p_{1}+R p_{2}\right)}\right],
\end{gathered}
$$


where $\quad \mathbf{H}_{3}(\alpha, \beta, \gamma ; x, y)=\sum_{m, n=0}^{\infty} \frac{(\alpha)_{2 m+n}(\beta)_{n}}{(\gamma)_{m+n} m ! n !} x^{m} y^{n}$ is one of the twenty convergent confluent hypergeometric series of order two (Horn function), and $(\alpha)_{n}$ is the Pochhammer symbol such that $(a)_{0}=1$ and $(a)_{k+1}=(a+k)(a)_{k}$ for any positive integer $k$ [14].

By taking into consideration both Eqs. 10, 11 and the Cochran's theorem [15], the PDF of the span estimator from Eq. 9 converges asymptotically to the the ratio of two correlated Gamma random variables PDF (the ratio of two quadratics). Moreover, the degrees of freedom $n_{1}$ and $n_{2}$ are set to $N[m /(m+1)]$ and $N$ (the number of secondary data), respectively.

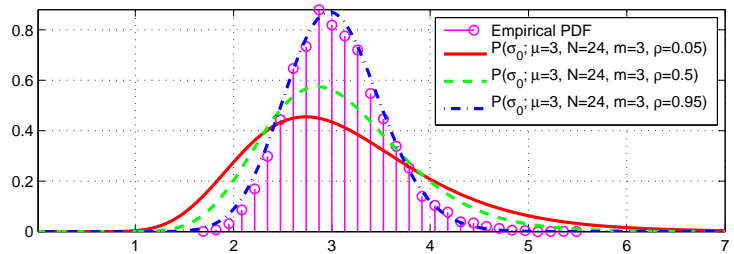

Fig. 1. Ratio PDF of two correlated Gamma random variables (Eq. 11 for different $\rho$ and the empirical PDF of simulated $\sigma_{0}$ in Gaussian clutter

Fig. 11illustrates the behavior of the $\sigma_{0}$ PDF with respect to the normalized correlation coefficient $\rho$. The PDF parameters are set according to the processing illustrated in Sect. IV] namely $N=24, m=3, \mu_{1}=10, \mu_{2}=1$. Notice that when the normalized correlation coefficient approaches to 1 , the PDF tends to a Dirac.

A Monte Carlo simulation has been represented in Fig 1 also. 5000 samples of $\sigma_{0}$ were obtained by computing $5000 \times$ 24 samples draw from a zero-mean multivariate circular complex Gaussian distribution with a covariance matrix selected from the real POLSAR data. The span of the selected covariance matrix equal 3. One can observe the good correspondence between the empirical PDF of simulated $\sigma_{0}$ and the PDF derived in Eq. 11 for $\rho=0.95$.

TABLE I

EMPIRICAL MEAN AND VARIANCE OF THE $\sigma_{0}$ ESTIMATOR FROM EQ.9 AND THE THEIR EXPECTED VALUES FOR SIMULATED GAUSSIAN CLUTTER.

\begin{tabular}{||c||c|c|c|c||}
\hline \hline \multirow{2}{*}{ Boxcar } & \multicolumn{2}{|c|}{ Expected } & \multicolumn{2}{c||}{ Empirical } \\
\cline { 4 - 5 } & Mean & Variance & Mean & Variance \\
\hline \hline $3 \times 3$ & \multirow{3}{*}{3} & \multirow{3}{*}{0} & 3.42 & 1.99 \\
\cline { 4 - 5 } & & 3.13 & 0.51 \\
\hline $5 \times 5$ & & 3.04 & 0.22 \\
\hline $7 \times 7$ & & & 3.03 & 0.13 \\
\hline $9 \times 9$ & & & \\
\hline \hline
\end{tabular}

Using the same parameters as in the previous Monte Carlo simulation, Table [ illustrates the behavior of the empirical mean and variance of the proposed $\sigma_{0}$ in Gaussian clutter (e.g. in homogeneous regions). By using 24 up to 48 secondary data, the estimation bias is negligible and the empirical variance is close to zero.

\section{B. The $\sigma_{0}$ test}

In this section we propose to show how the estimator from Eq. 9 is linked with a binary hypothesis testing problem, also:
- under the null hypothesis $H_{0}$, the observed target vector $\mathbf{k}=\sqrt{\xi} \cdot \zeta$ belongs to the SIRV clutter $\zeta \sim \mathcal{N}(0,[T])$ with normalized texture,

- under the alternative hypothesis $H_{1}$, the primary target vector $\mathbf{k}=\sqrt{\tau} \cdot \mathbf{z}$ belongs to the SIRV clutter $\mathbf{z} \sim$ $\mathcal{N}(0,[M])$ with normalized covariance matrix.

From the operational point of view, the proposed detector is a classical constant false alarm rate detector with current pixel as primary data, and with the local boxcar neighborhood around it as secondary data.

The Neyman-Pearson optimal detector is given by the following likelihood ratio test (LRT):

$$
\Lambda(\mathbf{k})=\frac{p_{\mathbf{k}}\left(\mathbf{k} / H_{1}\right)}{p_{\mathbf{k}}\left(\mathbf{k} / H_{0}\right)} \underset{H_{0}}{\stackrel{H_{1}}{\gtrless}} \lambda .
$$

After expressing the PDF under each hypothesis, it results that:

$$
\Lambda(\mathbf{k})=\frac{\frac{1}{\pi^{m} \operatorname{det}\{[M]\} \tau^{m}} \exp \left(-\frac{\mathbf{k}^{\dagger}[M]^{-1} \mathbf{k}}{\tau}\right)}{\frac{1}{\pi^{m} \operatorname{det}\{[T]\} \xi^{m}} \exp \left(-\frac{\mathbf{k}^{\dagger}[T]^{-1} \mathbf{k}}{\xi}\right)} \underset{H_{0}}{\stackrel{H_{1}}{H_{0}}} \lambda .
$$

By plugging into the LRT the ML texture estimators from Eqs. 5 and 8 we obtain:

$$
\Lambda(\mathbf{k})=\frac{\operatorname{det}\{[T]\}}{\operatorname{det}\{[M]\}}\left(\frac{\mathbf{k}^{\dagger}[T]^{-1} \mathbf{k}}{\mathbf{k}^{\dagger}[M]^{-1} \mathbf{k}}\right)^{m} \underset{H_{0}}{\stackrel{H_{1}}{\gtrless}} \lambda .
$$

Next, we assume the ratio of determinants is a deterministic quantity and we denote it by $\alpha$. This is an approximation, since in practice the ratio of determinants is also computed using the ML estimators of the respective covariance matrix with $N$ secondary data. Finally, by replacing the known covariances by their ML estimates the generalized LRT is:

$$
\Lambda(\mathbf{k})=\alpha \widehat{\sigma}_{0}^{-m} \underset{H_{0}}{\stackrel{H_{1}}{\gtrless}} \lambda .
$$

As $\alpha$ appears as a deterministic quantity only, it is possible to use the PDF derived in Sect. IV-A to set the decision threshold $\lambda$ for a specific false alarm probability.

\section{RESULTS AND DISCUSSIONS}

The high resolution POLSAR data set, illustrated in Fig. 2 was acquired by the ONERA RAMSES system over Toulouse, France with a mean incidence angle of $50^{\circ}$. It represents a fully polarimetric (monostatic mode) X-band acquisition with a spatial resolution of approximately $50 \mathrm{~cm}$ in range and azimuth. In the upper part of the image one can observe the CNES buildings.

Fig. 5-(a),(b),(c) presents the three SIRV parameters which completely describe the POLSAR data set: the total power, the normalized texture and the normalized covariance matrix. The $5 \times 5$ boxcar neighborhood has been selected for illustration, hence 24 secondary samples and 1 primary data.

Fig. 3 presents the zoom over the red rectangle from Fig. 5. (a), where a narrow diplane target was previously detected. Fig. 3-(a),(b),(c) shows the FP-PWF texture, the SCM-PWF normalized texture, and the proposed span estimator $\widehat{\sigma_{0}}$, respectively. For comparison, the Multi-look PWF (MPWF) has been illustrated in Fig. 3-(d). The proposed estimator exhibits 


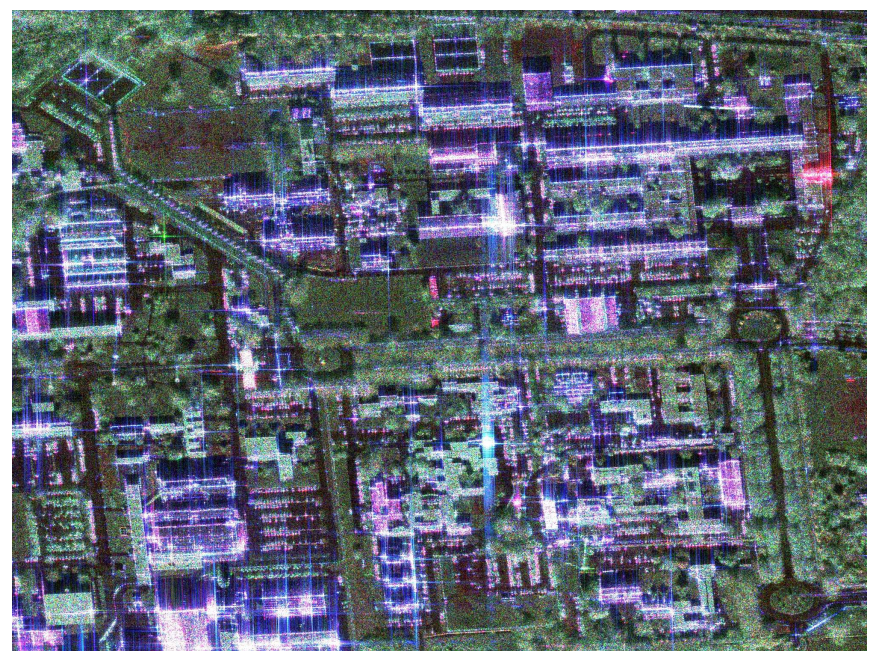

Fig. 2. Toulouse, RAMSES POLSAR data, X-band, $1500 \times 2000$ pixels: amplitude color composition of the target vector elements $k_{1}-k_{3}-k_{2}$.

better performances in terms of spatial resolution preservation than the MPWF span estimator: the ring effect (two large dips on a spatial profile near the boundaries of a pointwise target [16]) is reduced.

Finally, Fig. 4 illustrates the detection map obtain using the LRT from Eq. 15 with 25 secondary and one primary data. The detection threshold has been obtained by Monte Carlo integration of the PDF from Eq. 11 with a false alarm probability set to $P_{f a}=10^{-3}$ in each pixel. Note that the PDF integration for such a small $P_{f a}$ is quite time consuming and fast numerical approximations need to be investigated in the future for going to an operational level. This detection map can be interpreted as follows:

- heterogeneous clutter areas, represented in red, revel dense urban areas, which exhibit fewer dominant scatterers within the resolution cell. Over these areas, according to the hypotheses test from Sect. IV, it is better to estimate clutter parameters using the normalized covariance SIRV model.

- homogeneous clutter areas, represented in blue, where the normalized texture model is better.

Concerning the validation of our results, the generalized LRT is known to be asymptotically uniformly most powerful according to the Neyman-Pearson lemma [17]. This "optimality" holds provided the ML estimators plugged into the LRT are consistent, which is the case for our study [11], [12].

\section{CONCLUSIONS}

This paper presented a new estimation scheme for optimally deriving clutter parameters with high resolution POLSAR images. The heterogeneous clutter in POLSAR data was described by the SIRV model. Three estimators were introduced for describing the high resolution POLSAR data set: the span, the normalized texture and the speckle normalized covariance matrix. The asymptotic distribution of the new span estimator has been established. The estimation bias on homogeneous regions have been assessed also by Monte Carlo simulations.
Based on these issues, a novel test has been introduced for selecting the most appropriate model for POLSAR heterogeneous clutter described by SIRVs.

This work has many interesting perspectives. We believe that this paper contributes toward the description and the analysis of heterogeneous clutter over scenes exhibiting complex polarimetric signatures. Firstly, the exact texture normalization condition for the PWF-SCM estimator has been derived in Sect. [under the SIRV clutter hypothesis. A novel estimation / detection strategy has been proposed which can be used with conventional boxcar neighborhoods directly. Finally, the proposed estimation scheme can be extended to other multidimensional SAR techniques using the covariance matrix descriptor, such as the following: repeat-pass interferometry, polarimetric interferometry, or multifrequency polarimetry.

\section{ACKNOWLEDGMENT}

The authors would like to thank Dr. S. Zozor and Dr. F. Chatelain (GIPSA-lab, France) for the very fruitful discussions and advices. The authors would also like to thank Dr. C. Tison (CNES, France) for providing the high-resolution POLSAR images over Toulouse.

\section{REFERENCES}

[1] L. M. Novak and M. C. Burl, "Optimal speckle reduction in polarimetric SAR imagery," IEEE Transactions on Aerospace and Electronic Systems, vol. 26, no. 2, pp. 293-305, 1990.

[2] A. Lopes and F. Sery, "Optimal speckle reduction for the product model in multilook polarimetric SAR imagery and the Wishart distribution," IEEE Transactions on Geoscience and Remote Sensing, vol. 35, no. 3, pp. 632-647, 1997

[3] L. M. Novak, M. C. Burl, and W. W. Irving, "Optimal polarimetric processing for enhanced target detection," IEEE Transactions on Aerospace and Electronic Systems, vol. 29, no. 1, pp. 234-244, 1993.

[4] G. Liu, S. Huang, A. Torre, and F. Rubertone, "The Multilook Polarimetric Whitening Filter (MPWF) for intensity speckle reduction in polarimetric SAR images," IEEE Transactions on Geoscience and Remote Sensing, vol. 36, no. 3, pp. 1016-1020, 1998.

[5] G. Vasile, J.-P. Ovarlez, F. Pascal, and C. Tison, "Coherency matrix estimation of heterogeneous clutter in high resolution polarimetric SAR images," IEEE Transactions on Geoscience and Remote Sensing, vol. 48, no. 4, pp. 1809-1826, 2010.

[6] B. Picinbono, "Spherically invariant and compound Gaussian stochastic processes," IEEE Transactions on Information Theory, vol. 16, no. 1, pp. 77-79, 1970.

[7] K. Yao, "A representation theorem and its applications to sphericallyinvariant random processes," IEEE Transactions on Information Theory, vol. 19 , no. 5, pp. 600-608, 1973.

[8] S. Zozor and C. Vignat, "Some results on the denoising problem in the elliptically distributed context," IEEE Transactions on Signal Processing, vol. 58, no. 1, pp. 134-150, 2010.

[9] F. Gini and M. V. Greco, "Covariance matrix estimation for CFAR detection in correlated heavy tailed clutter," Signal Processing, vol. 82, no. 12 , pp. 1847-1859, 2002.

[10] E. Conte, A. DeMaio, and G. Ricci, "Recursive estimation of the covariance matrix of a compound-Gaussian process and its application to adaptive CFAR detection," IEEE Transactions on Image Processing, vol. 50, no. 8, pp. 1908-1915, 2002.

[11] F. Pascal, Y. Chitour, J.-P. Ovarlez, P. Forster, and P. Larzabal, "Covariance structure maximum-likelihood estimates in compound Gaussian noise: existence and algorithm analysis," IEEE Transactions on Signal Processing, vol. 56, no. 1, pp. 34-48, 2008.

[12] F. Pascal, P. Forster, J.-P. Ovarlez, and P. Larzabal, "Performance analysis of covariance matrix estimates in impulsive noise," IEEE Transactions on Signal Processing, vol. 56, no. 6, pp. 2206-2216, 2008.

[13] F. Chatelain, J. Y. Tourneret, and J. Inglada, "Change detection in multisensor SAR images using bivariate gamma distributions," IEEE Transactions on Image Processing, vol. 17, no. 3, pp. 249-258, 2008. 
[14] A. Erdlyi, W. Magnus, F. Oberhettinger, and F. Tricomi, Higher Transcendental Functions. New York: Krieger, 1981, vol. 1.

[15] W. G. Cochran, "The distribution of quadratic forms in a normal system, with applications to the analysis of covariance," Mathematical Proceedings of the Cambridge Philosophical Society, vol. 30, no. 2, pp. 178-191, 1934.

[16] J. S. Lee, S. R. Cloude, K. P. Papathanassiou, M. R. Grunes, and I. H. Woodhouse, "Speckle filtering and coherence estimation of polarimetric SAR interferometry data for forest applications," IEEE Transactions on Geoscience and Remote Sensing, vol. 41, no. 10, pp. 2254-2263, 2003.

[17] L. L. Scharf, Statistical Signal Processing: Detection, Estimation, and Time Series Analysis. Addison-Wesley, Inc., 1991.

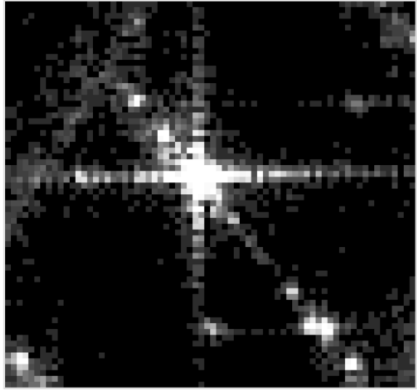

(a)

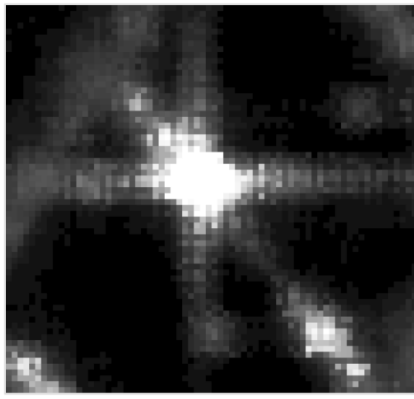

(c)

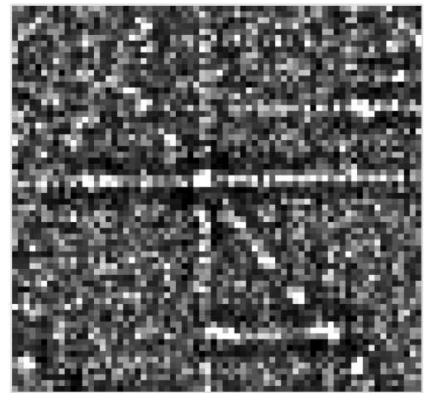

(b)

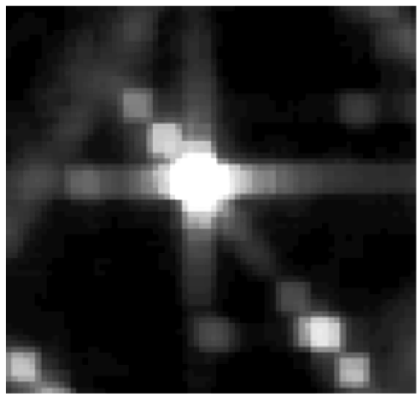

(d)
Fig. 3. Toulouse, RAMSES POLSAR data, X-band, $50 \times 50$ pixels, zoom image: (a) FP-PWF texture, (b) SCM-PWF normalized texture, (c) span estimated using $\widehat{\sigma_{0}}$ from Eq. 9 and (d) SCM-MPWF span.

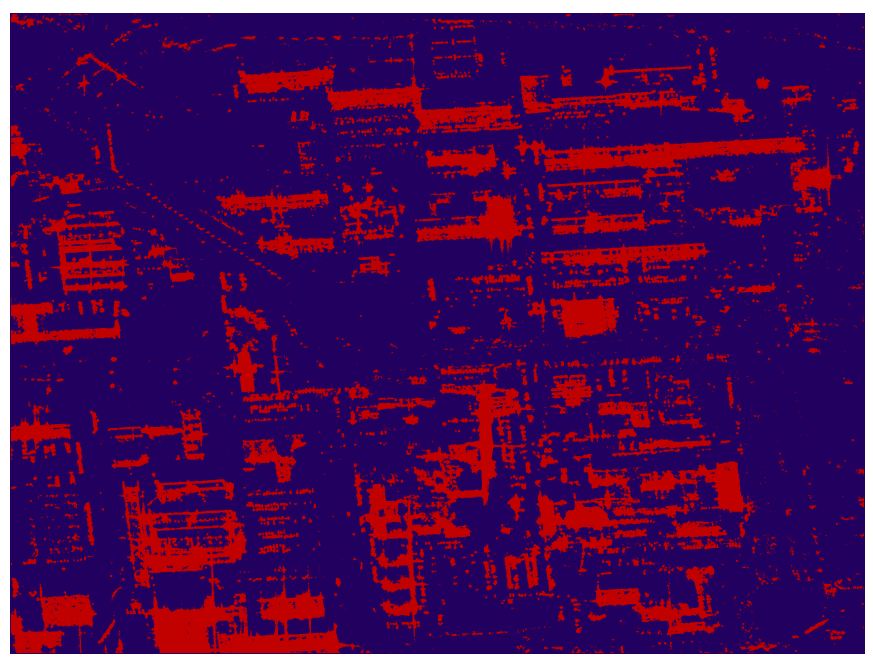

Fig. 4. Toulouse, RAMSES POLSAR data, X-band, $1500 \times 2000$ pixels: LRT detection map at $P_{f a}=10^{-3}$ (SIRV with normalized texture in blue and SIRV with normalized covariance in red).

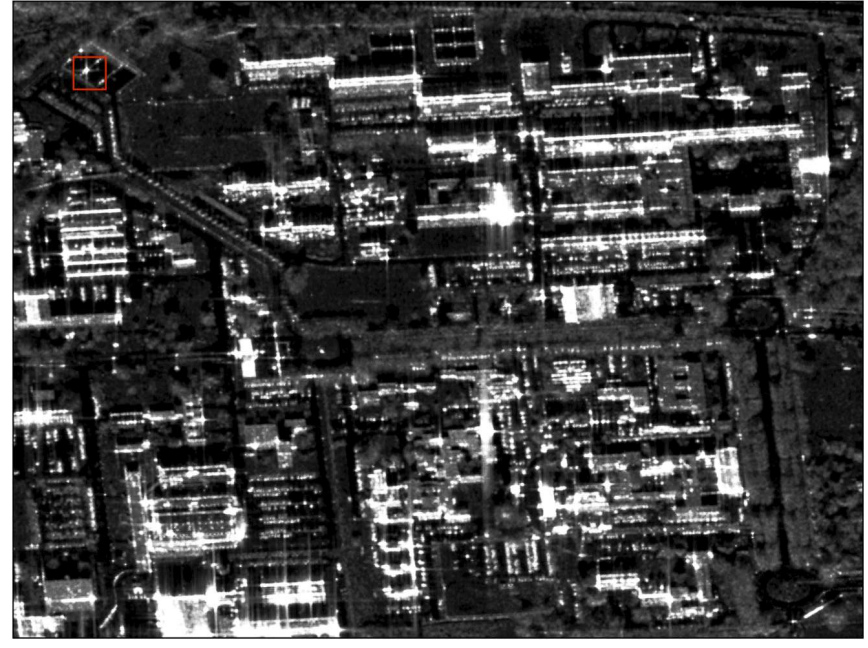

(a)

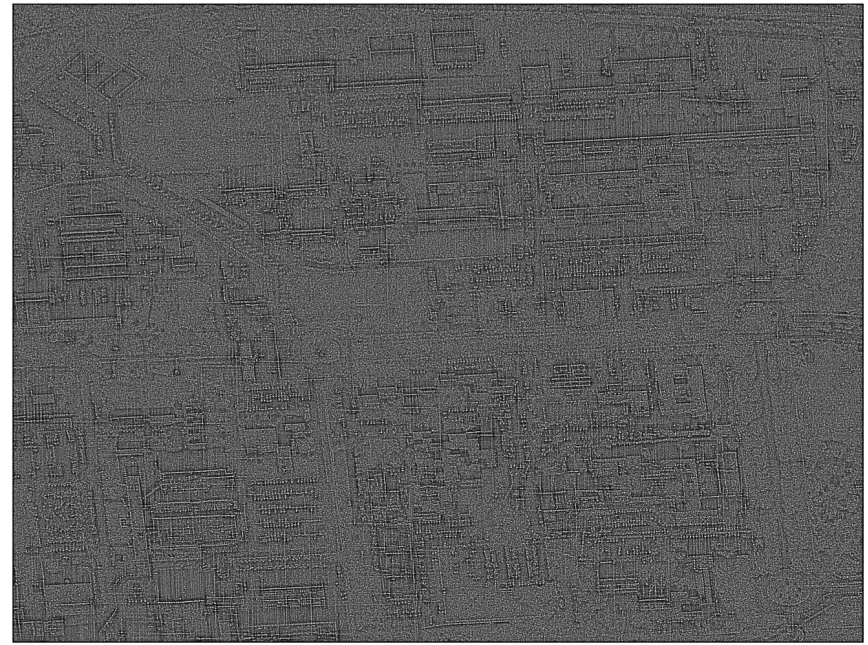

(b)

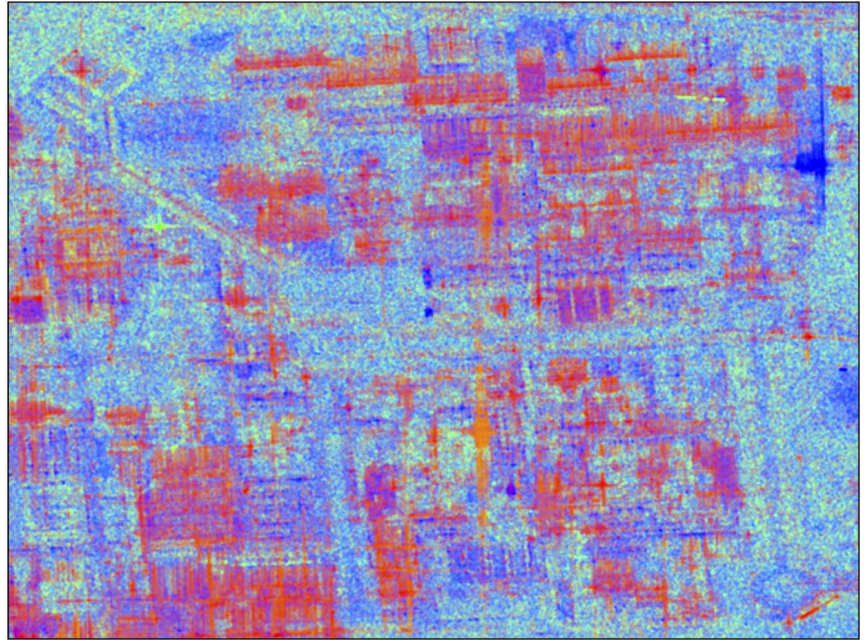

(c)

Fig. 5. Toulouse, RAMSES POLSAR data, X-band, $1500 \times 2000$ pixels: (a) span estimated using $\widehat{\sigma_{0}}$ from Eq. 9 (b) normalized texture $\xi$, and (c) color composition of the normalized coherency diagonal elements $[M]_{11}-[M]_{33}-$ $[M]_{22}$. 